\title{
城市地下综合管线信息系统数据更新模式探讨
}

\author{
王英红 \\ 义乌市勘测设计研究院 \\ DOI:10.32629/gmsm.v2i2.101
}

[摘 要] 近年来,在社会经济稳步发展的背景下,我国城市建设工程事业呈现了较为快速的发展势态。对于城市地下综合管线 工程来说,属于城市建设工程中非常重要的一个环节。为了确保城市地下综合管线工作质量及安全性的提高, 有必要注重其信 息系统的构建。本课题在分析城市地下综合管线信息系统数据管理的目标及内容的基础上,进一步提出其系统数据更新模式 关键技术及技术的实现步骤流程, 以期提高城市地下综合管线工程的整体工作效率及质量。

[关键词] 城市地下综合管线工程；信息系统数据；关键技术；工作质量

城市地下综合管线信息系统建设工作, 属于城市基础设 施工程之一, 该项工作存在规模大、密集的显著特点, 尤其是 在城市现代化建设过程中, 其工作负荷大, 管理项目多, 为了 满足现代化城市快速发展的需求, 有必要优化城市地下综合 管线信息系统建设工作, 合理利用现代化技术, 融合科学的 方法, 实现信息数据共享及加快数字化城市建设 ${ }^{[1]}$ 。由此可 见, 从现代化城市发展角度考虑, 本课题围绕 “城市地下综合 管线信息系统数据更新模式” 进行探讨具备一定的价值意 义。

1 城市地下综合管线信息系统数据管理目标及内容 分析

1.1 管理目标

对于城市地下综合管线信息系统来说, 主要是以全面普 查建成区地下管线空间分布及相应的属性情况为基础, 根据 城市的规划思路, 构建具有时效性及现代化特点的地下管线 综合数据系统。系统把地下管线信息通过数字化的方式在数 据系统库当中存储, 使以市为单位的管线数据得到综合有效 的管理。总结起来, 其具体管理目标包括: (1) 将探测规程与 普查流程作为基础, 构建将探测成果作为目标的计算机监理 机制以及相关实施规范标准; (2)利用以 GIS 技术为基础的 数据库管理系统, 支持外业勘测、内业成图以及管理信息系 统构建等服务; (3) 使城市地下综合管线工作实现网络化管 理及计算机化管理, 确保信息数据具备动态更新功能; (4) 实现对城市规划建设及管理提供技术方案的支持; (5) 使政府 及管线权属单位能够具备决策、指挥以及管理等科学依据, 为 城市现代化发展提供多元化服务, 促进城市可持续发展 ${ }^{[2]}$ 。

\section{2 管理内容}

将城市地下管线数据标准作为依据, 构建完善的地下管 线数据库, 使城市规划具备有效的决策依据。总结起来, 城市 地下综合管线信息系统数据管理的内容包括: (1) 构建完善 的标准体系。加强数据标准建设, 包括管线数据库标准、管 线数据入库标准等; 同时, 加强技术规范建设, 比如元数据规 范、代码编写规范以及界面设计规范等 ${ }^{[3]}$ 。(2) 构建完善的 空间数据库。整合城市管线普查成果数据、管线模型数据以
及基础地形图数据等, 然后根据相关规范要求, 构建空间数 据库, 为信息平台的应用提供有效支撑。(3)构建完善的系统 平台。涵盖地下管线数据监理成图子系统、 $\mathrm{CAD}$ 综合应用子 系统以及地下管线数据管理子系统等。

2 城市地下综合管线信息系统数据更新模式关键技术 分析

2. 1 以 ORACLE、 ESRI 以及 AutoCAD 为基础的应用

对于城市综合地下管线信息系统, 主要对供水、热力、 煤气、雨水、污水以及电力等管线实现集中可视化管理。由 于管理工作复杂、规模庞大, 因此需注重关键技术的合理应 用。以某城市地下综合管线信息系统数据更新模式所采用的 关键技术为例, 其中空间数据层使用了 Oracle $11 \mathrm{~g}+\mathrm{ArcSDE}$ 技术, 使基础的存储有效实现; 同时, 使用 ArcSDE 作为数据 服务器, 进而构建成数据管理层, 并通过. NET 开放语言和 ArcEngine 相结合, 构建系统数据管理层的应用, 经 SDE 使数 据访问有效实现, 并使数据库调用、查询以及统计等功能得 到有效实现; 此外, 通过 AutoCAD 技术的应用, 使电子报建功 能开发有效实现。

\section{2 跨越 CAD 及 GIS 平台的技术应用}

同时, 在该城市地下综合管线信息系统数据更新工作中, 通过跨平台技术的应用, 基于规划业务审批期间, 采取 AutoCAD 技术, 使 CAD 和 GIS 数据间的双向存取通道得到有 效拓展 ${ }^{[4]}$ 。并基于统一的大环境下, 使图属互动有效实现, 同 时基于图形、属性查询条件下, 均能够实现业务案卷的查询、 分析、统计以及业务办理等功能。

\section{3 面向服务架构的 SOA 技术}

此外, 对于面向服务的构建, 属于一种构架方法, 可以使 松耦合、互操作等业务服务有效创建, 且这些服务科在企业 内外部实现共享。面向服务架构利用 SOA 技术, 实现对不同 功能单元的服务, 并经服务间定义良好的接口与契约, 在接 口位置使用中立方式定义, 使系统实现统一标准的通信功能, 包括硬件平台功能操作、操作系统功能操作以及编程语言功 能操作等。

\section{3 技术实现步骤流程分析}


3.1 管线普查数据入库模式

在城市地下综合管线信息系统数据处理过程中, 管线普 查数据入库是非常重要的一个环节。在构建完成系统之后, 需针对外业探测标段验收成果进行入库处理。进行实时数据 管线库的构建, 以此视为管线信息化建设的基础部分。考虑 到管线数据的准确性实效性得到有效保障, 在相关管线业务 开展及数据更新工作过程中, 均需符合该系统的规范标准。

3.2 管线数据动态更新模式

对于管线工程竣工验收的数据更新, 也就是综合管线数 据库数据的更新, 主要方法有两种: 其一, 要素级更新方法; 其二, 范围级更新方法。其具体实现方法为:

3. 2.1 要素级更新方法。利用 $\mathrm{CAD}$ 辅助设计系统, 登陆 之后, 利用 $\mathrm{CAD}$ 展点功能叠加竣工测量后的点线表中间格式 数据, 进一步对目前的管线数据进行调用, 通过比较两组数 据, 然后结合用户的具体需求进行合理调整, 同时对数据状 态进行同步更新修改。之后, 对管线监理成图模块调用, 根据 相关规定进行核实, 通过句柄标识的方法记录错误数据, 经 句柄能够对 CAD 图面中提示用户修改的数据进行定位, 通过 多次核实修改, 在明确数据没有质量问题后, 将数据的相关 要素进行入库更新处理。

3.2 .2 范围级更新方法。该方法适合在大范围管线数据 动态更新处理工作中应用, 比如新增道路、道路改造等动态 数据。考虑到处理工作的便利, 针对大范围管线更新工作, 可以使用重新探测的方法, 使探测范围当中的全部管线实现 更新替代。

总之, 要素级更新方法适合解决管线数据动态更新问题, 具备效率高、咒余数据小以及维护便利等优势; 范围级更新 方法则适用大范围管线数据动态更新 ${ }^{[5]}$ 。需结合城市地下综 合管线信息系统实际工作需求, 合理选择管线数据动态更新 模式方法。

\section{3 地下管线数据监理成图系统及管理模式}

一方面, 对于地下管线数据监理成图系统, 使用了 $\mathrm{C} / \mathrm{S}$ 架构, 其工作任务为监理、检查地下综合管线数据; 比如: 数据的载入、监理, 元数据检查, 辅助纠错, 管线成图查询等。 利用地下管线数据监理成图系统, 能够在信息数据入库之前,
实现对管线点线表入库数据的检查, 使入库数据的准确性得 到有效保障。另一方面, 对于地下管线数据的管理模式, 同样 使用了 $\mathrm{C} / \mathrm{S}$ 架构, 涵盖系统调图、地图操作、数据加载以及 查询统计等功能; 主要管理任务为管线数据更新入库更新入 库管理, 利用 $\mathrm{CAD}$ 综合应用系统及上述提到的跨平台技术实 现综合管理。此外, 利用运营管理系统, 实现系统的安全管理, 比如用户的权限管理、角色管理等, 同时包括日志管理、配 置管理等。总之, 合理地利用这些管理模式方法, 能够使城市 地下综合管线信息系统得到全面、有效的管理,进一步确保 城市地下综合管线信息系统管理工作质量及安全性的提高。

\section{4 结语}

综上所述, 城市地下管线信息管理系统的建设是非常重 要的一项工作。首先, 需要明确城市地下管线信息管理系统 建设的目标及内容; 其次, 结合具体工程项目, 选择合理科学 的现代化关键技术, 包括: 以 ORACLE、ESRI 以及 AutoCAD 为 基础的技术应用、跨越 $\mathrm{CAD}$ 及 GIS 平台的技术应用以及面向 服务架构的 SOA 技术应用等, 使城市地下管线信息管理系统 的建设得到科学技术的有效支撑。此外, 还有必要选择合理 的管线普查数据入库模式与管线数据动态更新模式, 通过加 强对地下管线数据更新的管理, 使城市地下综合管线信息系 统建设整体工作的质量及安全性得到有效提高, 最终为城市 的健康、稳步发展奠定坚实的基础。

\section{[参考文献]}

[1]刘桥喜,熊伟,孙光辉,等.面向多源数据集成的城市地 下综合管廊安全运营与智慧管控研究 [J]. 地理信息世 界,2019,26(01):37-40.

[2]李芉,段雯,许高强.基于 DEMATEL 的综合管廊运维管 理风险因素研究[J].隧道建设(中英文),2019,39(01):31-39.

[3]安泽宇,郭旺.地下轨道交通和综合管廊协同建设相 关问题研究 [J]. 隧道建设(中英文),2019,39(01):130-138.

[4]邸海燕.城市综合管廊交叉节点受力性能研究 [J]. 山 东工业技术,2019,(04):112-113.

[5]陈丽.城市地下综合管廊运营阶段风险管理研究 [J]. 现代建筑电气,2019,10(01):48-52. 